\title{
Graphene on metals: A van der Waals density functional study
}

Vanin, Marco; Mortensen, Jens Jørgen; Kelkkanen, Kari André; García Lastra, Juan Maria; Thygesen, Kristian Sommer; Jacobsen, Karsten Wedel

Published in:

Physical Review B Condensed Matter

Link to article, DOI:

10.1103/PhysRevB.81.081408

Publication date:

2010

Document Version

Publisher's PDF, also known as Version of record

Link back to DTU Orbit

Citation (APA):

Vanin, M., Mortensen, J. J., Kelkkanen, K. A., García Lastra, J. M., Thygesen, K. S., \& Jacobsen, K. W. (2010). Graphene on metals: A van der Waals density functional study. Physical Review B Condensed Matter, 81(8), 081408. https://doi.org/10.1103/PhysRevB.81.081408

\section{General rights}

Copyright and moral rights for the publications made accessible in the public portal are retained by the authors and/or other copyright owners and it is a condition of accessing publications that users recognise and abide by the legal requirements associated with these rights.

- Users may download and print one copy of any publication from the public portal for the purpose of private study or research.

- You may not further distribute the material or use it for any profit-making activity or commercial gain

- You may freely distribute the URL identifying the publication in the public portal 


\title{
Graphene on metals: A van der Waals density functional study
}

\author{
M. Vanin, J. J. Mortensen, A. K. Kelkkanen, J. M. Garcia-Lastra, K. S. Thygesen, and K. W. Jacobsen \\ Center for Atomic-Scale Materials Design, Department of Physics, Technical University of Denmark, \\ DK-2800 Kongens Lyngby, Denmark
}

(Received 15 December 2009; revised manuscript received 1 February 2010; published 23 February 2010)

\begin{abstract}
We use density functional theory (DFT) with a recently developed van der Waals density functional (vdW$\mathrm{DF}$ ) to study the adsorption of graphene on $\mathrm{Co}, \mathrm{Ni}, \mathrm{Pd}, \mathrm{Ag}, \mathrm{Au}, \mathrm{Cu}, \mathrm{Pt}$, and $\mathrm{Al}(111)$ surfaces. In contrast to the local-density approximation (LDA) which predicts relatively strong binding for Ni,Co, and Pd, the vdW-DF predicts weak binding for all metals and metal-graphene distances in the range 3.40-3.72 $\AA$. At these distances the graphene band structure as calculated with DFT and the many-body $G_{0} W_{0}$ method is basically unaffected by the substrate, in particular there is no opening of a band gap at the $K$ point.
\end{abstract}

DOI: 10.1103/PhysRevB.81.081408

PACS number(s): 73.20.Hb, 71.15.Mb, 71.15.Nc

The recently reported synthesis of graphene, ${ }^{1}$ a single layer of graphite, on top of a $\mathrm{SiO}_{2}$ substrate has renewed the interest for this unique material. The uniqueness of this twodimensional (2D) crystal is mainly due to its very peculiar band structure, with the $\pi$ and $\pi^{*}$ bands showing linear dispersion around the Fermi level where they touch in a single point. The great variety of physics and chemistry which derives from this electronic structure makes graphene very attractive for a range of applications. In particular, its high stability and good conductivity under ambient conditions makes it an interesting candidate for future nanoscale electronics. ${ }^{2}$ In this perspective, the interaction of graphene with metallic contacts plays a fundamental role. Moreover, catalytic growth of graphene on transition metal surfaces from carbon containing gases has become a standard way to obtain high quality graphene samples. ${ }^{3-6}$ Nevertheless the nature of the metal-graphene chemical bond is still not well understood. ${ }^{3}$

The widely used density functional theory (DFT) with local and semilocal functionals for exchange and correlation usually provides an accurate description of covalent and ionic chemical bonds. On the other hand it fails to reproduce nonlocal dispersive forces, in particular van der Waals forces, which are important in weakly bonded materials such as graphite, molecular crystals, and many organic compounds. $^{7-9}$ It is also well known that the local-density approximation (LDA) tends to overbind systems where van der Waals interactions are important, while the generalized gradient approximations (GGAs) usually tend to underestimate the binding in these systems. In the case of graphene on metals many GGAs, contrary to experiments, predict no binding at all, and therefore most theoretical work on graphene-metal interfaces has relied on the LDA. In view of the fact that LDA in general cannot be considered a reliable approximation in nonhomogeneous systems such as surfaces and molecules, the graphene-metal interface clearly calls for new and improved functionals.

The interaction of graphene with the (111) surfaces of Co, $\mathrm{Ni}, \mathrm{Pd}, \mathrm{Ag}, \mathrm{Au}, \mathrm{Cu}, \mathrm{Pt}$, and $\mathrm{Al}$ was studied in Ref. 10 using the LDA approximation. The LDA results divide the metals into two classes: $\mathrm{Co}, \mathrm{Ni}$, and $\mathrm{Pd}$ which bind graphene strongly and $\mathrm{Ag}, \mathrm{Au}, \mathrm{Cu}, \mathrm{Pt}$, and $\mathrm{Al}$ which bind graphene weakly. In contrast the Perdew-Burke-Ernzerhof (PBE) approximation (Ref. 11) gives no binding of graphene at room temperature. ${ }^{12}$ This remarkable disagreement between the two most commonly used approximations of DFT might be related to the incorrect description of dispersion interactions in both of the functionals.

In this Rapid Communication we use the recently developed van der Waals density functional (vdW-DF) (Refs. 13 and 14) to investigate the nature of the bonding at the metalgraphene interface. The functional is explicitly constructed to include nonlocal dispersion interactions and has proved successful in several cases where standard functionals fail, such as rare gases, ${ }^{13}$ benzene dimers, ${ }^{15,16}$ graphite, ${ }^{17}$ polymers, ${ }^{18}$ DNA, ${ }^{19}$ and organic molecules on surfaces. ${ }^{20-22}$ Within the $\mathrm{vdW}-\mathrm{DF}$ approximation, the exchange-correlation energy is

$$
E_{x c}^{\mathrm{vdW}-\mathrm{DF}}=E_{x}^{\mathrm{revPBE}}+E_{c}^{\mathrm{LDA}}+E_{c}^{\mathrm{nl}},
$$

where $E_{x}^{\text {revPBE }}$ is the revPBE (Ref. 23) exchange energy, $E_{c}^{\mathrm{LDA}}$ is the LDA correlation energy, and $E_{c}^{\mathrm{nl}}$ is the nonlocal correction given by

$$
E_{c}^{\mathrm{nl}}=\frac{1}{2} \iint n\left(\mathbf{r}_{1}\right) n\left(\mathbf{r}_{2}\right) \phi\left(q_{1}, q_{2}, r_{12}\right) d \mathbf{r}_{1} d \mathbf{r}_{2},
$$

where $r_{12}=\left|\mathbf{r}_{1}-\mathbf{r}_{2}\right|$ and $q_{1}$ and $q_{2}$ are values of a universal function $q_{0}(n(\mathbf{r}),|\nabla n(\mathbf{r})|)$. Equation (2) is efficiently evaluated by factorizing the integration kernel $\phi$ and by using fast Fourier transform to compute the self-consistent potential as proposed in Ref. 24 and implemented in the real-space projector augmented wave GPAW code. ${ }^{25}$

In this Rapid Communication we consider graphene on $\mathrm{Co}, \mathrm{Ni}, \mathrm{Pd}, \mathrm{Ag}, \mathrm{Au}, \mathrm{Cu}, \mathrm{Pt}$, and $\mathrm{Al}$ metal (111) surfaces. We fix the atoms in the metal slabs at their experimental lattice parameters and relax the graphene sheet using the vdW-DF Hellmann-Feynman forces. We observe that the vdW-DF results do not change significantly if we fix the graphene lattice parameter to its optimized value and adjust the metals correspondingly. We use a $(6,6,1)$ and $(4,4,1)$ Monkhorst Pack k-point sampling, respectively, for the smaller $(\mathrm{Co}, \mathrm{Ni}$, and $\mathrm{Cu})$ and the larger $(\mathrm{Pd}, \mathrm{Ag}, \mathrm{Au}, \mathrm{Pt}$, and $\mathrm{Al}$ ) orthorhombic unit cells. The two different unit cells are needed in order to obtain commensurate structures without significant strain, as discussed in Ref. 10. The metal slabs are modeled with four atomic layers and a vacuum of $14 \AA$ in the direction normal to the surface; the grid spacing is $0.16 \AA$. The calculations 
TABLE I. Binding energies $\left(E_{b}\right)$ per carbon atom and binding distances $(d)$ of graphene on metal (111) surfaces. Fermi level shift $\Delta E_{\mathrm{F}}$ and charge transfer $\delta Q$ at the vdW-DF equilibrium separation. Negative (positive) $\Delta E_{\mathrm{F}}$ indicates $n(p)$-type doping. Negative (positive) $\delta Q$ indicates electron transfer to (from) the graphene layer. The charge transfer has been evaluated using the Bader scheme (Ref. 26). The revPBE functional yields no binding for all metals.

\begin{tabular}{|c|c|c|c|c|c|c|c|c|c|}
\hline & & Co & $\mathrm{Ni}$ & $\mathrm{Pd}$ & $\mathrm{Ag}$ & $\mathrm{Au}$ & $\mathrm{Cu}$ & $\mathrm{Pt}$ & $\mathrm{Al}$ \\
\hline \multirow[t]{4}{*}{ vdW-DF } & $d(\AA)$ & 3.40 & 3.50 & 3.50 & 3.55 & 3.57 & 3.58 & 3.67 & 3.72 \\
\hline & $E_{b}(\mathrm{meV})$ & 30 & 37 & 39 & 33 & 38 & 38 & 43 & 35 \\
\hline & $\Delta E_{\mathrm{F}}(\mathrm{eV})$ & -0.20 & 0.13 & 0.65 & -0.40 & 0.21 & -0.43 & 0.66 & -0.51 \\
\hline & $\delta Q\left(10^{-3} e\right)$ & -5.0 & -3.0 & +5.0 & -5.0 & +0.4 & -4.0 & +5.0 & -8.0 \\
\hline \multirow[t]{2}{*}{ LDA } & $d(\AA)$ & 2.08 & 2.08 & 2.33 & 3.32 & 3.35 & 3.21 & 3.25 & 3.46 \\
\hline & $E_{b}(\mathrm{meV})$ & 175 & 123 & 79 & 45 & 31 & 35 & 33 & 25 \\
\hline \multirow[t]{2}{*}{ Expt. } & $d(\AA)$ & $1.5-2.2^{\mathrm{a}}$ & $2.1^{b}$ & & & $5^{\mathrm{c}}$ & & $3.3^{\mathrm{d}}$ & \\
\hline & Hybridization & strong a & strong $\mathrm{e}$ & strong $\mathrm{f}$ & weak $\mathrm{g}$ & weak $^{c}$ & weak ${ }^{g}$ & weak ${ }^{d}$ & \\
\hline
\end{tabular}

${ }^{a}$ Ref. 27

${ }^{b}$ Ref. 28

${ }^{\text {cRef. }} 29$

${ }^{\mathrm{d}}$ Ref. 30

for $\mathrm{Ni}$ and $\mathrm{Co}$ surfaces are spin polarized: notice however that the nonlocal correlation [last term in Eq. (1)] is independent of spin. The calculated binding energies and distances for the relaxed structures are listed in Table I. The vdW-DF results show that the metal-graphene interaction is relatively similar across the different metals. This is in contrast with the LDA prediction of two separate classes of metalgraphene interfaces, as found in very good agreement with Ref. 10. Note that in the LDA calculations the graphene lattice parameter was fixed to its optimized LDA value. We also repeated the same calculations using the revPBE functional, and we obtained no binding for all the metals. Interestingly, for the systems that LDA finds to be weakly bonded (Ag, $\mathrm{Au}$, $\mathrm{Cu}, \mathrm{Pt}$, and $\mathrm{Al}$ ), the binding energies obtained with the vdW-DF are very similar to the LDA ones. Nevertheless the binding distances are systematically slightly larger in the vdW-DF case. In fact it has been reported that the vdW-DF functional usually produces equilibrium distances somewhat larger than experiments. ${ }^{20}$ In the case of $\mathrm{Co}, \mathrm{Ni}$, and $\mathrm{Pd}$, on the other hand, the relatively strong binding predicted by LDA is not found by the vdW-DF functional.

In order to analyze these results, we now focus on the interaction between graphene and $\mathrm{Ni}(111)$. Figure 1 shows the binding curves for graphene on the $\mathrm{Ni}(111)$ surface calculated with the LDA, revPBE, and vdW-DF functionals. The revPBE curve is positive at all distances, while the LDA curve shows a relatively deep minimum at $\sim 2 \AA$ consistent with previous LDA calculations. The vdW-DF result lies in between, following the revPBE curve at small separations and the LDA curve at larger separations, and it predicts a shallow minimum at $3.5 \AA$. Note that a metastable plateau is found by the revPBE functional around $2.5 \AA$.

In Fig. 2 we show the calculated band structure of graphene on $\mathrm{Ni}(111)$. The size of the dots indicate the weight of the corresponding Bloch eigenstate on the carbon $p_{z}$ orbitals with darker meaning larger weight. In free graphene, the carbon $p_{z}$ orbitals placed at $A$ sites $\left(p_{z}^{A}\right)$ are decoupled from the $p_{z}$ orbitals at $B$ sites $\left(p_{z}^{B}\right)$ at the Dirac point, thus
${ }^{\mathrm{e}}$ Ref. 31

${ }^{\text {fRef. }} 32$

gRef. 33

producing two degenerate states (see inset in Fig. 1 for the structure). Since the $A$ sites are located directly on top of $\mathrm{Ni}$ atoms at a close distance in the LDA calculation $(2.08 \AA)$, a strong hybridization between $p_{z}^{A}$ orbitals and $\mathrm{Ni}_{3 z-r}$ is observed, which gives rise to an unoccupied antibonding state $\sigma^{*}$ and two occupied bonding states $\sigma_{1}$ and $\sigma_{2}$. The LDA gaps for $\sigma^{*}-\sigma_{1}$ and $\sigma^{*}-\sigma_{2}$ are 2 and $4 \mathrm{eV}$, respectively. On the other hand, the $p_{z}^{B}$ orbitals (occupied in the spin-up channel and unoccupied in the spin-down one) hardly interact with $\mathrm{Ni} d$ states and therefore remain unmodified. The vdW-DF band structures (evaluated at the vdW-DF relaxed distance of $3.50 \AA$ ), on the other hand, resemble the free graphene, preserving the Dirac point and only shifting it up by $0.13 \mathrm{eV}$. A very similar behavior is found for $\mathrm{Co}$ and $\mathrm{Pd}$. For the remaining interfaces both the LDA and vdW-DF band structures resemble that of free-standing graphene with the Dirac point shifted with respect to the metal Fermi level. The Fermi level shifts and calculated charge transfer between the metal and graphene are summarized in Table I.

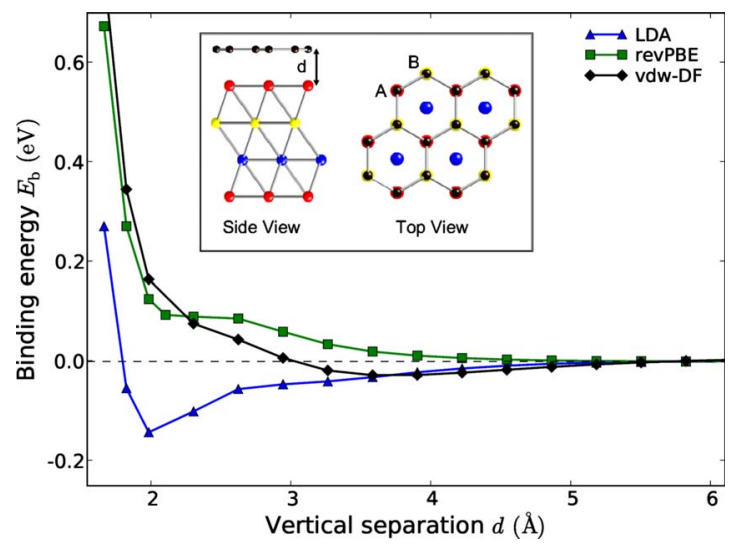

FIG. 1. (Color online) Binding energy $\left(E_{b}\right)$ per carbon atom of graphene on the $\mathrm{Ni}(111)$ surface calculated with LDA, revPBE, and vdW-DF functionals. The graphene is adsorbed in the top-fcc configuration (see inset). 

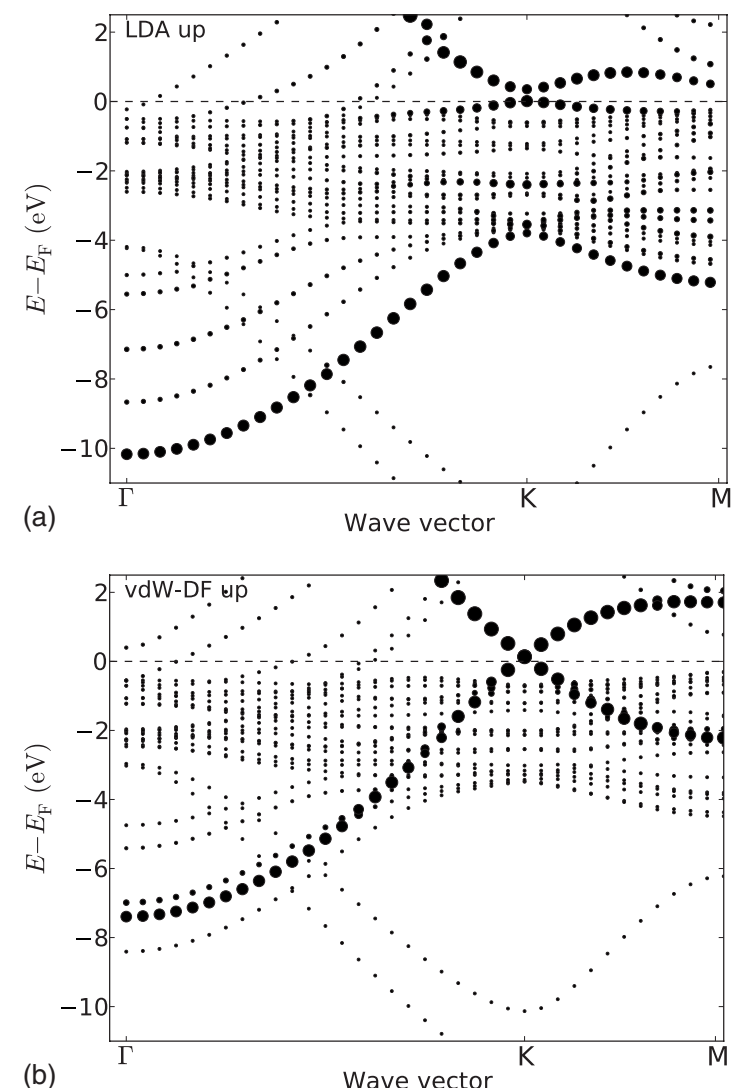

FIG. 2. LDA (top) and vdW-DF (bottom) band structures for graphene on $\mathrm{Ni}(111)$ in the top-fcc configuration. Larger dots represent larger weight of the carbon $p_{z}$ orbitals. Only one spin channel is shown, the other being almost identical.

Since LDA is known to underestimate band gaps significantly we have also performed $G_{0} W_{0}$ calculations for the graphene-Ni structures corresponding to the LDA and vdW-DF distances. ${ }^{34}$ In both cases we find no noteworthy difference between the $G_{0} W_{0}$ and DFT Kohn-Sham band structures close to the $K$ point.

Recent experimental work on the Ni/graphene interface is based on angle-resolved photoemission spectroscopy (ARPES). The ARPES band structures reveal a band gap at the graphene $K$ point thus suggesting some hybridization between the graphene and Ni orbitals. ${ }^{31,35}$ Earlier low-energy electron diffraction (LEED) measurements found a $\mathrm{Ni}$ graphene bond distance of $2.1 \AA{ }^{28}$ We note that both of these results are in line with the LDA calculations. On the other hand LDA is not expected to work well for highly inhomogeneous systems such as the interface structures investigated here. Similar experimental conclusions ${ }^{27}$ are found for the $\mathrm{Co} / \mathrm{graphene}$ interface, using scanning tunneling spectroscopy (STS) techniques. These results might indicate a difficulty for the present vdW-DF in describing systems with mixed bonding character, in line with the conclusions of Ref. 22. Moreover, results involving the application of the vdW-DF functional to metallic systems should be taken with care due to the choice of the response function employed in the construction of the vdW-DF. ${ }^{36} \mathrm{It}$ should also be noted that the present calculations are restricted to two specific interface configurations and hence do not take into account the variety of Moiré superstructures observed in experiments. Recent ARPES and LEED data is also available for the $\mathrm{Pt} /$ graphene interface ${ }^{30}$ which provides clear evidence of weak interaction between graphene and the substrate, with an estimated separation of $3.3 \AA$, in fair agreement with both LDA and our vdW-DF results. STS measurements for graphene on Pd (Ref. 32) show a band-gap opening of $\sim 0.3 \mathrm{eV}$, in contrast with both LDA and vdW-DF predictions. STS experiments on the Au/graphene interface, ${ }^{29}$ particularly relevant since most electronic contacts employ gold, demonstrate a weak interaction between $\mathrm{Au}$ and graphene, and a $p$-type doping in good agreement with both LDA and our vdW-DF results. We have summarized the available recent experimental data in the last two rows of Table I.

Figure 3 shows the total (full lines) and the exchange-only (dashed lines) binding energy curves for revPBE, PBE, and LDA in the case of graphene on $\mathrm{Cu}(111)$ (left panel) and on $\mathrm{Ni}(111)$ (right panel). The exchange-only energies are calculated without including the correlation energy term in the exchange-correlation functional and have been evaluated non-self-consistently. Clearly, the bonding for the physisorbed graphene on $\mathrm{Ag}, \mathrm{Au}, \mathrm{Cu}, \mathrm{Pt}$, and $\mathrm{Al}$ originates partially from the exchange term in the LDA $x c$-functional, as
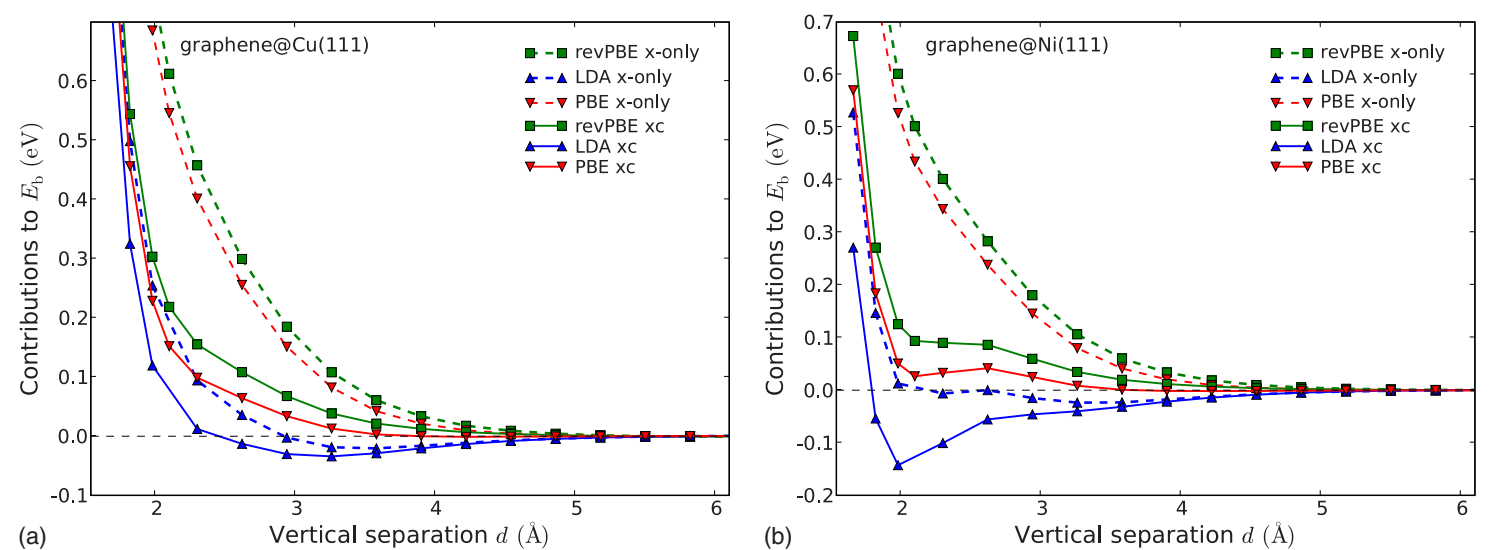

FIG. 3. (Color online) Decomposition of the binding energy $E_{b}$ into exchange-only contributions (dashed lines-only the correlation term is removed) and total binding energy (full lines) for different functionals. 
shown for $\mathrm{Cu}$ as an example in the left panel of Fig. 3. This is in principle incorrect since the van der Waals interaction is a purely nonlocal correlation effect. The weak bonding predicted by the vdW-DF functional, similar in magnitude to the LDA results, is produced by the correlation term instead, which is physically correct. Interestingly, this applies to the $\mathrm{Ni}$ /graphene system as well, as shown in the right panel of Fig. 3. The LDA exchange-only curve shows a broad and weak attractive contribution between 2 and $5 \AA$ with two local minima. We note that in a genuine covalent bond the exchange contribution to the binding energy is generally significantly larger than in this case. The revPBE and PBE exchange-only curves are repulsive at all separations for both systems. This is the typical behavior which is observed in van der Waals bonded dimers or organic molecules on surfaces. ${ }^{13,21}$

In conclusion we have performed DFT calculations of graphene adsorbed on different metal surfaces using the re- cently developed vdW-DF functional which explicitly includes nonlocal correlations. For $\mathrm{Ag}, \mathrm{Au}, \mathrm{Cu}, \mathrm{Pt}$, and $\mathrm{Al}$ both LDA and vdW-DF consistently predicts a weak binding. Similar weak binding is found for $\mathrm{Ni}$, $\mathrm{Co}$ and $\mathrm{Pd}$ with the vdW-DF where LDA on the other hand predicts stronger binding and significant hybridization between graphene and metal $d$ states. At the vdW-DF binding distances graphene's band structure was shown to be essentially unaffected by the substrate. This appears to be in conflict with LEED and ARPES measurements for graphene on $\mathrm{Ni}, \mathrm{Co}$, and $\mathrm{Pd}$ indicating that more work is needed in order to reconcile experiments and theory for the graphene-metal interface.

We thank Jens Nørskov and Bengt Lundqvist for useful discussions. The authors acknowledge support from the Danish Center for Scientific Computing through Grant No. HDW-1103-06. The Center for Atomic-scale Materials Design is sponsored by the Lundbeck Foundation.
${ }^{1}$ K. S. Novoselov et al., Science 306, 666 (2004).

${ }^{2}$ A. K. Geim and K. S. Novoselov, Nature Mater. 6, 183 (2007).

${ }^{3}$ J. Wintterlin and M.-L. Bocquet, Surf. Sci. 603, 1841 (2009).

${ }^{4}$ K. S. Kim et al., Nature 457, 706 (2009).

${ }^{5}$ J. Coraux et al., New J. Phys. 11, 039801 (2009).

${ }^{6}$ P. W. Sutter et al., Nature Mater. 7, 406 (2008).

${ }^{7}$ P. Hobzaet al., J. Comput. Chem. 16, 1315 (1995).

${ }^{8}$ S. Kristyán and P. Pulay, Chem. Phys. Lett. 229, 175 (1994).

${ }^{9}$ E. Ruiz et al., J. Am. Chem. Soc. 117, 1141 (1995).

${ }^{10}$ G. Giovannetti, P. A. Khomyakov, G. Brocks, V. M. Karpan, J. van den Brink, and P. J. Kelly, Phys. Rev. Lett. 101, 026803 (2008).

${ }^{11}$ J. P. Perdew, K. Burke, and M. Ernzerhof, Phys. Rev. Lett. 77, 3865 (1996).

${ }^{12}$ M. Fuentes-Cabrera, M. I. Baskes, A. V. Melechko, and M. L. Simpson, Phys. Rev. B 77, 035405 (2008).

${ }^{13}$ M. Dion, H. Rydberg, E. Schröder, D. C. Langreth, and B. I. Lundqvist, Phys. Rev. Lett. 92, 246401 (2004).

${ }^{14}$ M. Dion, H. Rydberg, E. Schröder, D. C. Langreth, and B. I. Lundqvist, Phys. Rev. Lett. 95, 109902 (2005).

${ }^{15}$ A. Puzder, M. Dion, and D. C. Langreth, J. Chem. Phys. 124, 164105 (2006).

${ }^{16}$ T. Thonhauser, A. Puzder, and D. C. Langreth, J. Chem. Phys. 124, 164106 (2006).

${ }^{17}$ E. Ziambaras, J. Kleis, E. Schröder, and P. Hyldgaard, Phys. Rev. B 76, 155425 (2007).

${ }^{18}$ J. Kleis, B. I. Lundqvist, D. C. Langreth, and E. Schröder, Phys. Rev. B 76, 100201 (2007).

${ }^{19}$ V. R. Cooper, T. Thonhauser, A. Puzder, E. Schröder, B. I. Lundqvist, and D. C. Langreth, J. Am. Chem. Soc. 130, 1304 (2008).

${ }^{20}$ D. C. Langreth, B. I. Lundqvist, S. D. Chakarova-Káck, V. R. Cooper, M. Dion, P. Hyldgaard, A. Kelkkanen, J. Kleis, L. Kong, S. Li, P. G. Moses, E. Murray, A. Puzder, H. Rydberg, E. Schróder, and T. Thonhauser, J. Phys.: Condens. Matter 21, 084203 (2009).

${ }^{21}$ P. G. Moses, J. J. Mortensen, B. I. Lundqvist, and J. K. Nørskov, J. Chem. Phys. 130, 104709 (2009).
${ }^{22}$ L. Romaner, D. Nabok, P. Puschnig, E. Zojer, and C. AmbroschDraxl, New J. Phys. 11, 053010 (2009).

${ }^{23}$ Y. K. Zhang and W. T. Yang, Phys. Rev. Lett. 80, 890 (1998).

${ }^{24}$ G. Román-Pérez and J. M. Soler, Phys. Rev. Lett. 103, 096102 (2009).

${ }^{25}$ J. J. Mortensen, L. B. Hansen, and K. W. Jacobsen, Phys. Rev. B 71, 035109 (2005).

${ }^{26}$ W. Tang, E. Sanville, and G. Henkelman, J. Phys.: Condens. Matter 21, 084204 (2009).

${ }^{27}$ D. Eom, D. Prezzi, K. T. Rim, H. Zhou, M. Lefenfeld, S. Xiao, C. Nuckolls, M. S. Hybertsen, T. F. Heinz, and G. W. Flynn, Nano Lett. 9, 2844 (2009).

${ }^{28}$ Y. Gamo, A. Nagashima, M. Wakabayashi, M. Terai, and C. Oshima, Surf. Sci. 374, 61 (1997).

${ }^{29}$ Z. Klusek, P. Dabrowski, P. Kowalczyk, W. Kozlowski, W. Olejniczak, P. Blake, M. Szybowicz, and T. Runka, Appl. Phys. Lett. 95, 113114 (2009).

${ }^{30}$ P. Sutter, J. T. Sadowski, and E. Sutter, Phys. Rev. B 80, 245411 (2009).

${ }^{31}$ A. Varykhalov, J. Sanchez-Barriga, A. M. Shikin, C. Biswas, E. Vescovo, A. Rybkin, D. Marchenko, and O. Rader, Phys. Rev. Lett. 101, 157601 (2008).

${ }^{32}$ S.-Y. Kwon, C. V. Ciobanu, V. Petrova, V. B. Shenoy, J. Bareo, V. Gambin, I. Petrov, S. Kodambaka, Nano Lett. 9, 3985 (2009).

${ }^{33}$ A. M. Shikin, V. K. Adamchuka, and K. H. Rieder, Phys. Solid State 51, 2390 (2009).

${ }^{34}$ The $G_{0} W_{0}$ calculations were performed with the YAMBO code. ${ }^{37}$ We included 100 empty bands (corresponding to $40 \mathrm{eV}$ above the Fermi level), a $12 \times 12 k$ point for the simple rhombohedral unit cell, and a plasmon frequency of 1 Hartree.

${ }^{35}$ A. Grüneis and D. V. Vyalikh, Phys. Rev. B 77, 193401 (2008).

${ }^{36}$ T. Thonhauser, V. R. Cooper, S. Li, A. Puzder, P. Hyldgaard, and D. C. Langreth, Phys. Rev. B 76, 125112 (2007).

${ }^{37}$ A. Marini, C. Hogan, M. Grüning, and D. Varsano, Comput. Phys. Commun. 180, 1392 (2009). 